\title{
Reflets
}

Revue ontaroise d'intervention sociale et communautaire

\section{Le profil démographique et les déterminants de la santé des francophones en Ontario}

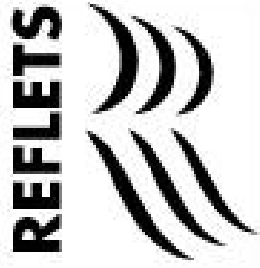

\section{Louise Picard et Janine Charland}

Volume 5, numéro 2, automne 1999

La santé des francophones de l’Ontario

URI : https://id.erudit.org/iderudit/026269ar

DOI : https://doi.org/10.7202/026269ar

Aller au sommaire du numéro

Éditeur(s)

Reflets : Revue ontaroise d'intervention sociale et communautaire

ISSN

1203-4576 (imprimé)

1712-8498 (numérique)

Découvrir la revue

Citer cet article

Picard, L. \& Charland, J. (1999). Le profil démographique et les déterminants de la santé des francophones en Ontario. Reflets, 5(2), 44-63.

https://doi.org/10.7202/026269ar

Tous droits réservés (C) Reflets : Revue ontaroise d'intervention sociale et communautaire, 1999
Ce document est protégé par la loi sur le droit d'auteur. L'utilisation des services d'Érudit (y compris la reproduction) est assujettie à sa politique d'utilisation que vous pouvez consulter en ligne.

https://apropos.erudit.org/fr/usagers/politique-dutilisation/ 


\title{
Le profil démographique et les déterminants de la santé des francophones en Ontario
}

\author{
L ouise P icard et Janine C harland
}

\section{Introduction}

«A fin de mieux cerner

l'état de santé des

francophones à l'aube de l'an 2000, il est important de comprendre leur contexte sodal. »
D es recherches antérieures sur l'état de santé des 0 ntariens et O ntariennes ont démontré des différences importantes entre les francophoneset les non- francophones (D ewit et al. 1995). Plusieurs auteurs confirment que les inégalités sociales sont liées aux différences quant à l'état de santé des gens (C omité consultatif fédéral provincial territorial sur la santé de la population, 1996) ${ }^{1}$.

A fin de mieux cerner l'état de santé des francophonesà l'aube de l'an 2000, il est important de comprendre leur contexte social. En effet, plusieurs variables, tels l'al phabétisme, I'éducation ou le revenu affectent directement et indirectement la santé des personnes. Ainsi, dans un effort de compréhension du contexte social danslequel évoluent lesFrancophones, cet article cherchera à résumer les traits principaux qui caractérisent cette société, à partir des analyses démographiquesmenées par l'O ffice desaffaires francophones et le Programme de recherche, éducation et développement du M inistère de santé de l'O ntario. C es analyses s'appuient sur les données les plus récentes de Statistique $C$ anada, extraites du R ecensement canadien de 1996. U n profil plusélaboré se trouve dans le rapport provincial, intitulé $R$ apport sur la santé des francophones en 0 ntario (1999). Les lecteurs sont invités à 
consulter le rapport intégral pour en savoir plus long sur la méthodologie utilisée, les définitions techniques, etc.

$L$ 'O ntario est la province canadienne qui regroupe le plus grand nombre de francophones vivant hors Q uébec. La population francophone représente le groupe minoritai re le plus important en O ntario. Par ailleurs, la L oi sur les services en français (1986) garantit la prestation de services en français dans toutes les collectivités où les francophones représentent plus de $10 \%$ de la population ou dans les centres urbains où on en dénombre au moins 5000 . Ainsi, la planification de services de santé et l'application de stratégies de promotion de la santé en 0 ntario requièrent une compréhension approfondie des déterminants de la santé de la population ontarienne, y compris la collectivité francophone.

Plusieurs études réalisées dansle passé ont identifié différentes caractéristiques démographiques et de santé de la population franco-ontarienne. $C$ es rapports antérieurs ( $O$ ffice des affaires francophones; Statistique Canada, 1996) ont révélé que la population francophone est plus âgée, moins scolarisée et qu'elle a des revenus et des taux d'alphabétisme moins élevés. Est-ce que ces différences persistent toujours en 1996? LaTable ronde sur la santé de la population et la promotion de la santé a clairement énoncé qu'afin d'améliorer la santé des $C$ anadiens, il est essentiel d'agir sur toute la gamme des déterminants de la santé (Santé Canada, 1996). U ne mise à jour du profil de la population francophone s'impose donc comme point de départ.

\section{Profil démographique}

Q ui sont lesfrancophones en 0 ntario et combien sommes-nous? Q uels ont été les principaux changements depuis le dernier recensement de 1991?

\section{Croissance et répartition de la population}

O n comptait 542340 francophones en O ntario, en 1996, soit $5,0 \%$ de la population de la province. $C$ e pourcentage représente 
une faible diminution par rapport au $R$ ecensement de 1991, alors que les francophones représentaient $5,4 \%$ de la population ontarienne (O ffice des affai res francophones et Statistique $C$ anada, 1996). $M$ algré une diminution de la proportion de francophones au sein de la population de l'O ntario, le nombre réel de francophones est demeuré relativement stable, soit une réduction de 1500 francophones échelonnée sur une période de dix ans, de 1986 à 1996. Ainsi, la diminution proportionnelle est attribuable, non pastellement à une bai sse des effectifs, mais à un accroissement de la population ontarienne dans son ensemble. Par conséquent, en matière de santé, les besoins de la population francophone demeurent constants et il faut chercher à les combler.

C ela dit, on note un changement de la répartition régionale de la population francophone au sein de la province (Figure 1). De 1991 à 1996, le N ord-Est a vu sa population francophone décroître de 8730 habitants, alors que la région Est en accueillait 9540 de

«En 1996, plus des deux tiers des francophones vivaient dans les régions de l'E st et du N ord-E st. » plus. En 1996, plus des deux tiers des francophones vivaient dans les régions de l'Est et du Nord-Est. C'est la région de l'Est qui compte le plus grand nombre de francophones, soit plus de 200 000 . Toutefois, le $\mathrm{N}$ ord-Est est la région où la proportion de francophones en fonction de la population globale est la plusélevée, soit environ le quart de la population (Tableau 1).

\section{Figure 1 - Distribution régionale de la population francophone}

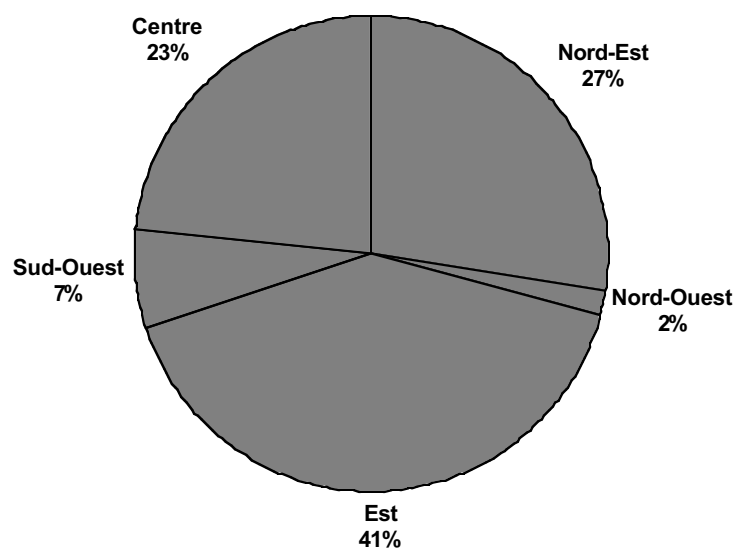

Source : Statistique C anada, R ecensement 1996, 2A , O A F 


\section{Tableau 1 - Représentation régionale de la population francophone}

\begin{tabular}{|c|c|c|}
\hline & $\mathbf{N}^{*}$ & $\begin{array}{c}\% \text { de pop. } \\
\text { totale }\end{array}$ \\
\hline Ontario & 542340 & $5,0 \%$ \\
\hline Est & 221100 & $15,0 \%$ \\
\hline Centre & 126650 & $1,8 \%$ \\
\hline Sud-Ouest & 35870 & $2,5 \%$ \\
\hline Nord-Est & 148955 & $25,6 \%$ \\
\hline Nord-Ouest & 9760 & $4,0 \%$ \\
\hline
\end{tabular}

Au niveau provincial, une plusgrande proportion de francophones vit en milieu rural. C e résultat peut s'expliquer par le fait que la majorité des francophones vivent dans les régions de l'Est et du $\mathrm{N}$ ord-Est, lesquelles sont plus rurales que les autres régions de la province. En effet, si nousétudions les données de plus près, nous constatons que, dans chacune de ces régions, les francophones ont plus tendance à vivre en région urbaine, comparativement à la population globale de la région (Figure 2).

\section{Figure 2 - Population rurale francophone et population rurale totale de I'Ontario, selon la région (Concentration $<1000$ et densité < à 400 au $\mathbf{k m}^{2}$ )}

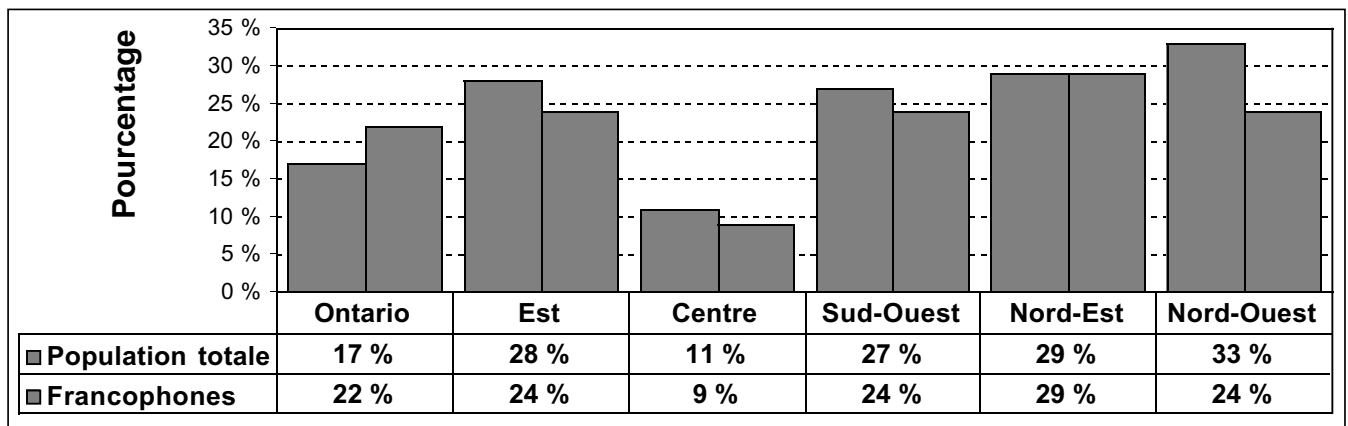

Source : Statistique C anada, R ecensement 1996, 2A , O A F 
«D eux francophones sur trois sont nés en 0 ntario. »

« N otons aussi que le pourcentage de francophones nés en 0 ntario diminue avec l'âge. »

\section{Immigration et groupes minoritaires}

Entre 1991 et 1996, 27800 francophonesont immigré en 0 ntario. Trois francophones sur cinq venaient du Q uébec et un sur cinq était originaire de l'extérieur du $\mathrm{C}$ anada. Seulement $6 \%$ des francophones sont nés à l'extérieur du $\mathrm{C}$ anada comparativement à $27 \%$ pour l'ensemble de la population de l'O ntario (Tableau 2). D eux francophones sur trois sont nés en 0 ntario. C'est dans le $\mathrm{N}$ ord-Est que la proportion de francophones nés en $\mathrm{O}$ ntario est la plus élevée, et dans le $C$ entre, qu'elle est la plus faible. Près du tiers des francophones du C entre et du $\mathrm{N}$ ord- $\mathrm{O}$ uest proviennent du Q uébec. N otons aussi que le pourcentage de francophones nés en 0 ntario diminue avec l'âge. En effet, près de $85 \%$ des francophones de 0 à 19 ans sont nés en 0 ntario, comparativement à $55 \%$ des francophones de 65 ans et plus.

Tableau 2 - Lieu de naissance

\begin{tabular}{|l|r|r|r|r|}
\hline & Ontario & Québec & $\begin{array}{c}\text { Autres } \\
\text { prov. }\end{array}$ & $\begin{array}{c}\text { A } \\
\text { l'extérieur } \\
\text { du Canada }\end{array}$ \\
\hline Ontario & $65,7 \%$ & $23,2 \%$ & $5,6 \%$ & $5,5 \%$ \\
\hline Est & $68,5 \%$ & $24,2 \%$ & $3,5 \%$ & $3,8 \%$ \\
\hline Centre & $42,0 \%$ & $31,2 \%$ & $12,1 \%$ & $14,7 \%$ \\
\hline Sud-Ouest & $65,6 \%$ & $20,9 \%$ & $7,8 \%$ & $5,7 \%$ \\
\hline Nord-Est & $82,0 \%$ & $15,3 \%$ & $2,2 \%$ & $0,5 \%$ \\
\hline Nord-Ouest & $51,7 \%$ & $30,8 \%$ & $15,7 \%$ & $1,9 \%$ \\
\hline Source: Statistique Canada, Recensement 1996, 2B, OAF \\
\hline
\end{tabular}

En 1996, on dénombrait 29000 francophones appartenant à des groupes minoritaires visibles, soit $6 \%$ de la population francophone totale. C es francophones vivaient principalement dans la communauté urbaine de Toronto et dans la région d'O ttawaC arleton, où ils représentaient, respectivement, $23 \%$ et $9 \%$ des collectivités francophones de ces régions (Tableau 3 ). Près des trois quarts (77\%) des francophones appartenant à une minorité visible sont nés à l'extérieur du pays. 


\section{Tableau 3 - Proportion de la population francophone appartenant à une minorité visible*}

\begin{tabular}{|l|r|}
\hline Régions & Pourcentage \\
\hline Ontario & $\mathbf{5 , 6} \%$ \\
\hline Est & $5,4 \%$ \\
\hline Centre & $12,7 \%$ \\
\hline Sud-Ouest & $4,4 \%$ \\
\hline Nord-Est & $0,3 \%$ \\
\hline Nord-Ouest & $0,5 \%$ \\
\hline Municipalité de la communauté & $22,5 \%$ \\
\hline urbaine de Toronto & $18,0 \%$ \\
\hline Région du Grand Toronto & $8.9 \%$ \\
\hline Région d'Ottawa-Carleton & $0,5 \%$ \\
\hline Municipalité régionale de Sudbury & S $\%$ \\
\hline Source : Statistique Canada, Recensement $1996,2 B$, OAF \\
\hline
\end{tabular}

* M inorité visible : fait partie des minorités visibles toute personne qui n' est pas de race blanche, à l'ex ception des A utochtones.

\section{Les groupes d'âge}

La population francophone demeure plus âgée que l'ensemble de la population ontarienne. Les francophones sont sur-représentés dans les groupes d'âge moyen et d'âge mûr, et sous représentés dans les catégories des plus jeunes (Figure 3). M ême si le vieillissement affecte l'ensemble de la population ontarienne, la population francophone demeure généralement plus âgée que

«Entre 1991 et 1996, le pourcentage de francophones de 65 ans et plus est passé de 12 $\%$ à $14 \%$ à l'échelle de la province » celle-ci. Entre 1991 et 1996, le pourcentage de francophones de 65 ans et plus est passé de $12 \%$ à $14 \%$ à l'échelle de la province. $D$ 'ailleurs, toutes les régions ont connu une croissance de la population des aînés francophones durant cette période. Cette caractéristique a une profonde influence sur l'état de santé des francophones et sur leurs besoins en matière de services de santé. 


\section{Figure 3 - Population francophone et population totale de l'Ontario selon l'âge}

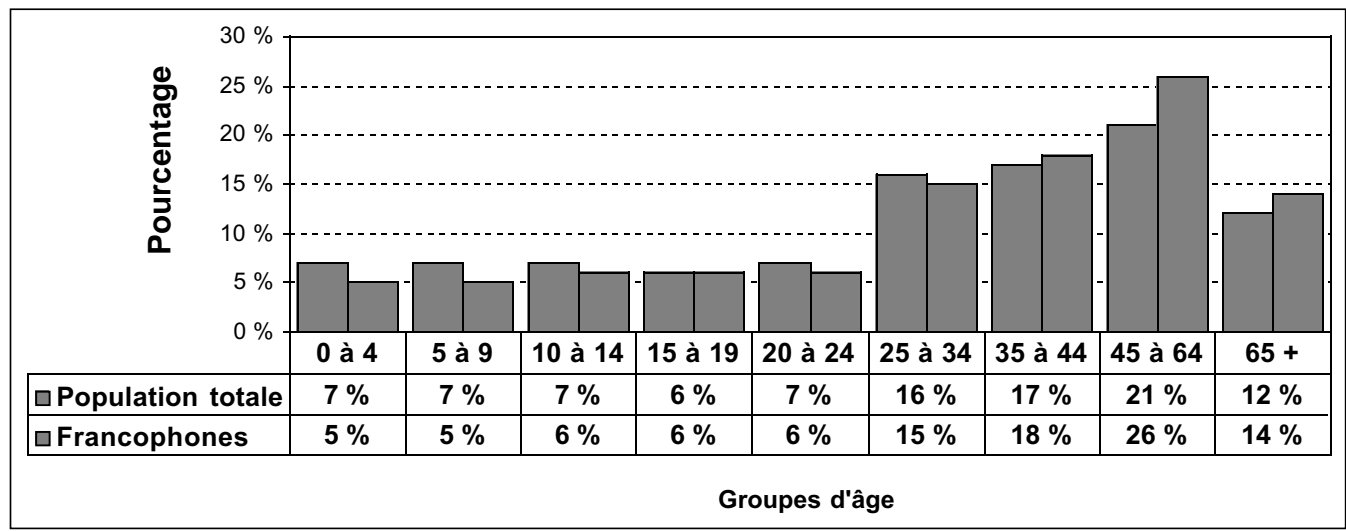

Source : Statistique C anada, R ecensement 1996, 2A , 0 A F

Au chapitre des catégories d'âge, nous observons aussi certaines différences régionales. L'Est et le $\mathrm{N}$ ord-Est sont les régions affichant le pourcentage le plus élevé de jeunes francophones de 14 ans ou moins (18\%), alors que le Sud0 uest a la plus faible proportion (10\%). D ans cette même région, le pourcentage de francophones de 65 ans et plus (22\%) est nettement plus élevé que celui des autres régions de la province.

Dans le recensement, une mesure intéressante liée aux groupes d'âge est le quotient de dépendance. C ette mesure est le rapport entre, d'une part, le nombre de jeunes (14 ans ou moins) ou de personnes âgées (65 ans et plus) pris séparément et, d'autre part, la population dite «active», soit les 15 à 64 ans qui sont en âge de participer au marché de travail. Ainsi, un quotient de dépendance élevé peut signifier un risque d'augmentation du fardeau fiscal pesant sur les jeunes, si on maintient les services, y comprisles services de santé, dansl'état actuel. 
Les données de 1996 montrent que le quotient de dépendance des jeunes francophones est inférieur à celui de l'ensemble de la population de la province et ce, dans toutes les régions. Par conséquent, les francophones sont plus susceptibles, en raison de leur répartition dans les diverses catégories d'âge, de participer au marché du travail. Toutefois, si nous examinons le quotient de dépendance relatif aux personnes âgées, nous pouvons raisonnablement envisager, dans un avenir rapproché, une hausse de celui-ci, compte tenu du vieillissement de la population francophone.

\section{Conservation de la langue française}

La conservation de la langue française est un sujet important et controversé dans la communauté francophone en 0 ntario. Plusieurs rapports récents sonnent l'alarme sur l'assimilation des francophones. Le recensement de 1996 présente un aperçu de ce phénomène en mesurant le niveau de conservation de la langue à partir de la langue parlée le plus souvent à la maison. De 1991 à 1996, le niveau de conservation du françaisen 0 ntario a diminué de $2 \%$. Le $\mathrm{N}$ ord- $\mathrm{O}$ uest est la région accusant la plus forte diminution, soit $6 \%$ en cinq ans.

«À l'échelle de la

province, trois

francophones sur anq utilisent le français le plus souvent, comme langue parlée à la maison (59\%). Le niveau de conservation du français varie selon les régions»
À l'échelle de la province, troisfrancophones sur cinq utilisent le françaisle plussouvent, comme langue parlée à la mai son (59\%). Le niveau de conservation du français varie selon les régions. La proportion de francophones parlant le français le plus souvent à la maison est plus élevée dans l'Est (71 \%) et le N ord-Est (69\%), ce qui n'est pas surprenant puisque ce sont les régions à forte concentration de francophones.

La proportion de jeunes francophones qui utilisent le français le plus souvent à la maison diminue avec l'âge (Figure 4). La baisse la plus importante survient durant la période de 15 à 24 ans et, on note également une baisse importante entre les âges de 5 à 14 ans. 


\section{Figure 4 - Conservation de la langue française, selon l'âge - population francophone}

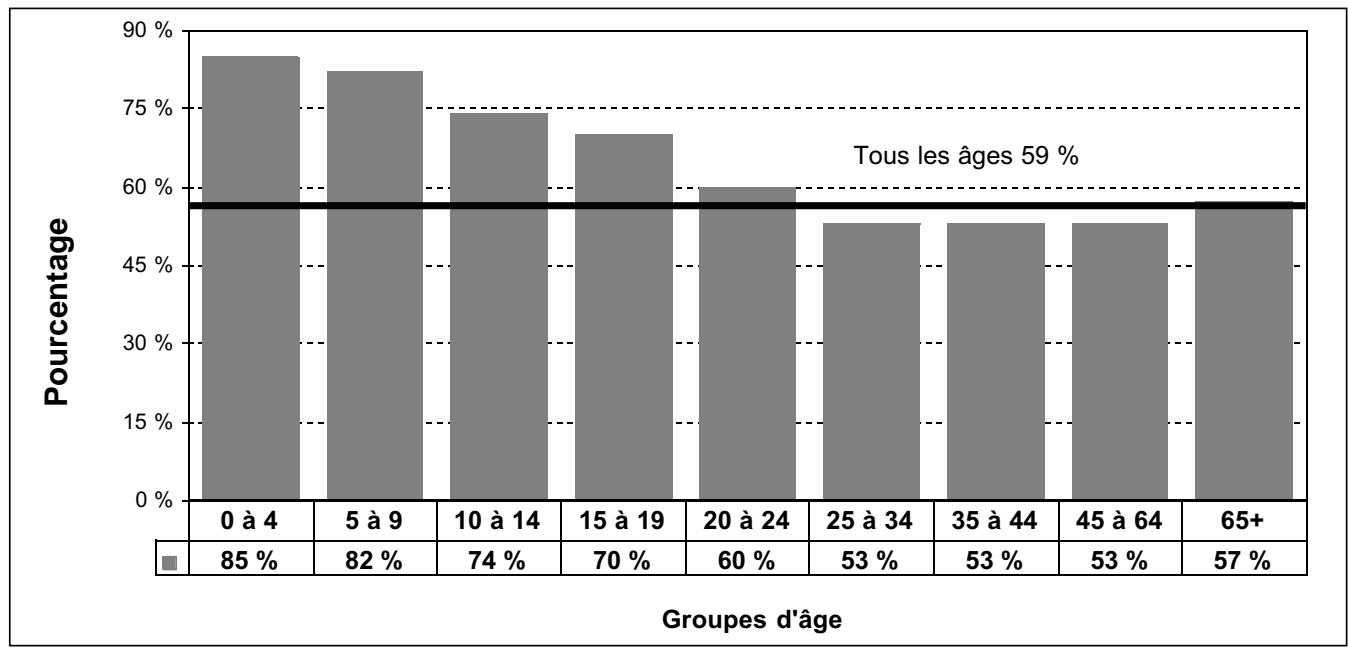

Source : Statistique C anada, R ecensement 1996, 2A, 0 A F

\section{Environnement social}

Pour améliorer la santé de la population, il faut comprendre l'influence qu'exercent les déterminants de la santé ou, si l'on préfère, ce qui, dans l'environnement social, fait qu'une personne est et demeure en bonne santé. C ette section aborde quelques-uns des déterminants particulièrement importants pour la société franco-ontarienne : la scolarité, la situation familiale, I'al phabétisme, I'emploi et le revenu. Il est important de souligner au départ qu'il existe des liens entre ces déterminants (e.g. entre le niveau de scolarité et le revenu) et que cela ajoute à la complexité de leurs effets sur la population (Zöller et Lesso, 1998).

Plusieurs auteurs établissent un lien entre les inégalités sociales et les différences dans l'état de santé des gens (Z öllner 
«En 0 ntario, la population francophone est généralement moins scolariséé que l'ensemble de la population provinciale »

«C e sont les francophones plus âgés qui sont nettement moins scolarisés que leurs homologues ontariens. » et Lesso, 1998).Voyons comment les francophones se situent à ce chapitre.

\section{Scolarisation}

La santé d'une population est affectée par son niveau de scolarité. D es études démontrent que plus le niveau de scolarité est élevé, meilleure est la perception de son état de santé, plus les limitations d'activités et la perte de journées de travail diminuent (Zöllner et Lesso, 1998).

En 0 ntario, la population francophone est généralement moins scolarisée que l'ensemble de la population provinciale. 0 n note ainsi qu'une plus faible proportion de francophones ( $12 \%$ ) possède un diplôme universitaire comparativement à la population totale (15\%) et qu'une plus forte proportion n'a pas complété la neuvième année ( $15 \%$ par rapport à $10 \%)$. C ependant, une analyse par groupe d'âge suggère que ces écarts s'amenuisent (Figure 5). En effet, chez les jeunes adultes de 20 à 24 ans et de 25 à 34 ans, la différence entre la population francophone et l'ensemble de la population de la province est mince. Ce sont les francophones plus âgésqui sont nettement moinsscolarisésque leurs homologues ontariens.

\section{Figure 5 - Population détenant au moins un baccalauréat, selon l'âge francophone et population totale de l'Ontario}

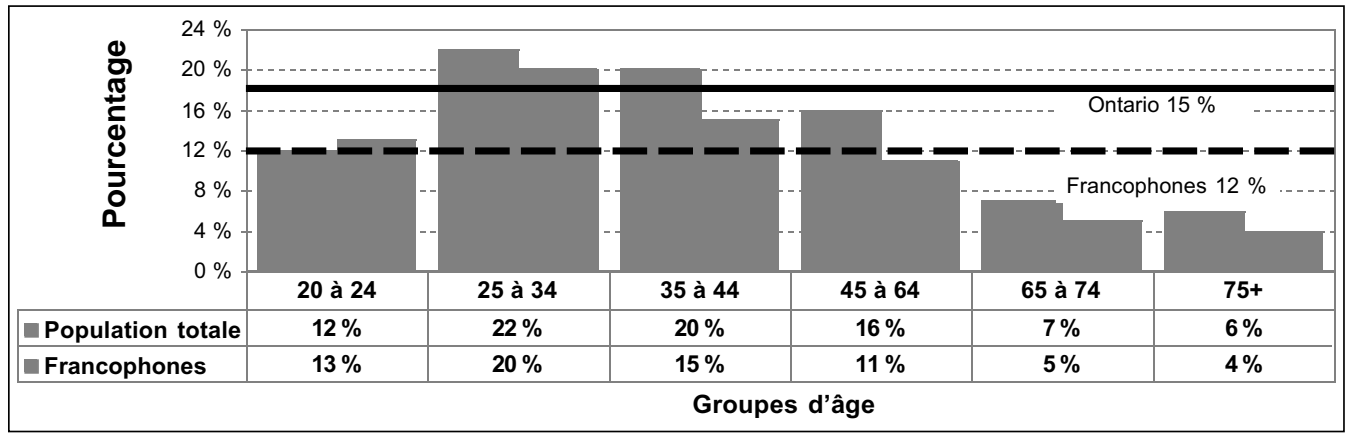

Source : Statistique C anada, R ecensement 1996, 2A , 0 A F 
O n a également noté l'existence de différences régionales. Ainsi, les francophones de l'Est et du Sud de la province sont généralement plus scolarisés que ceux du $\mathrm{N}$ ord et du Sud- $\mathrm{O}$ uest. (Figure 6).

\section{Figure 6 - Niveau de scolarité, selon la région - population francophone}

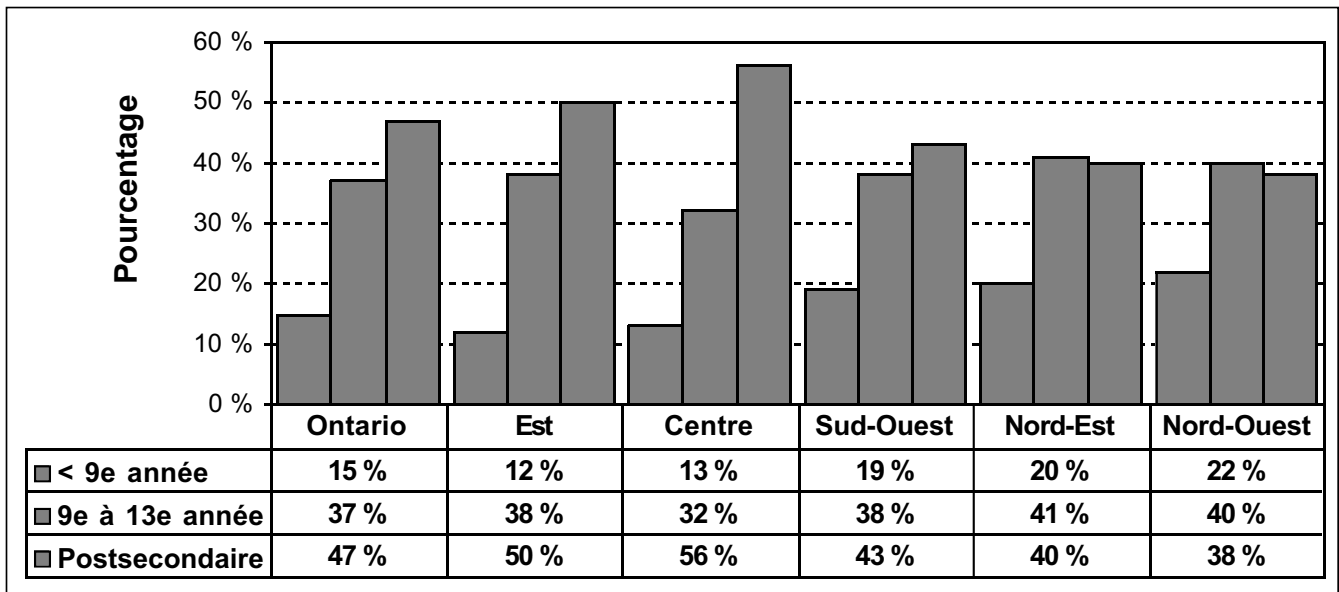

Source : Statistique C anada, R ecensement 1996, 2A , 0 A F

\section{Alphabétisme}

L'alphabétisme est étroitement lié à la santé. Les personnes qui possèdent de bonnes capacités de lecture et d'écriture obtiennent des emplois plus satisfaisants, touchent des revenus plus élevés et ont une meilleure qualité de vie (Comité consultatif fédéral provincial territorial sur al santé de la population, 1996). À l'inverse, de piètres capacités de lecture peuvent avoir une incidence sur des tâches aussi simples que la lecture de la posologie de médicaments (Shalla et Schellenberg, 1998). C ela dit, l'alphabétisme n'est plus défini comme jadisen fonction du niveau de scolarité, mais par les écarts de niveaux de compétences en lecture (Tableau 4) (Statistique C anada et Secrétariat national à 
I'alphabétisation, 1996). Les résultats suivants des francophones proviennent de l'Enquête internationale sur l'al phabétisation des adultes, menée en 1994 (G arceau, 1998).

Tableau 4 - Interprétation des niveaux d'alphabétisme

\begin{tabular}{|l|l|}
\hline \multicolumn{1}{|c|}{ Niveaux } & \multicolumn{1}{c|}{ Grille d'appréciation } \\
\hline $\begin{array}{l}\text { Niveau 1 } \\
\text { (0 à 225) }\end{array}$ & $\begin{array}{l}\text { Ces gens ont beaucoup de difficulté à } \\
\text { lire et admettent qu'ils ont un } \\
\text { problème }\end{array}$ \\
\hline $\begin{array}{l}\text { Niveau 2 } \\
\text { (226 à 275) }\end{array}$ & $\begin{array}{l}\text { Ces gens savent lire, mais ne lisent pas } \\
\text { bien. Ils sont capables de comprendre } \\
\text { des documents simples et clairement } \\
\text { présentés. }\end{array}$ \\
\hline $\begin{array}{l}\text { Niveau 3 } \\
\mathbf{( 2 7 6} \text { à 325) }\end{array}$ & $\begin{array}{l}\text { Ces gens lisent bien, mais peuvent } \\
\text { avoir de la difficulté à effectuer des } \\
\text { tâches plus complexes. }\end{array}$ \\
\hline $\begin{array}{l}\text { Niveau 4/5 } \\
\text { (326 à 500) }\end{array}$ & $\begin{array}{l}\text { Ces gens sont capables de satisfaire la } \\
\text { plupart des exigences de lecture. }\end{array}$ \\
\hline $\begin{array}{l}\text { Source : Statistique Canada, 1994 } \\
\text { Fiche technique tirée du document Lire l'avenir : un portrait de } \\
\text { l'alphabétisme au Canada }\end{array}$ \\
\hline
\end{tabular}

Les francophones ont, dans I'ensemble, de plus faibles capacités de lecture que les anglophones (Figure 7). C es plus faibles taux d'alphabétisme de la population francophone suscitent des inquiétudes depuis fort longtemps (Garceau 1998). Les différences de taux d'alphabétisme entre les franco phones et les anglophones peuvent, en grande partie, être associées à l'accès à l'enseignement dans leur langue maternelle. Les francophones qui font maintenant partie du groupe des aînés n'ont pas eu accès à l'enseignement primaire ou secondaire dans leur langue maternelle en 0 ntario; quant aux francophones d'âge moyen, ilsont dû fréquenter desécoles secondaires privées pour pouvoir faire leurs études en français. 


\section{Figure 7 - Répartition des capacités de lecture à l'échelle de compréhension des textes suivis, selon la langue du test - francophones et anglophones}

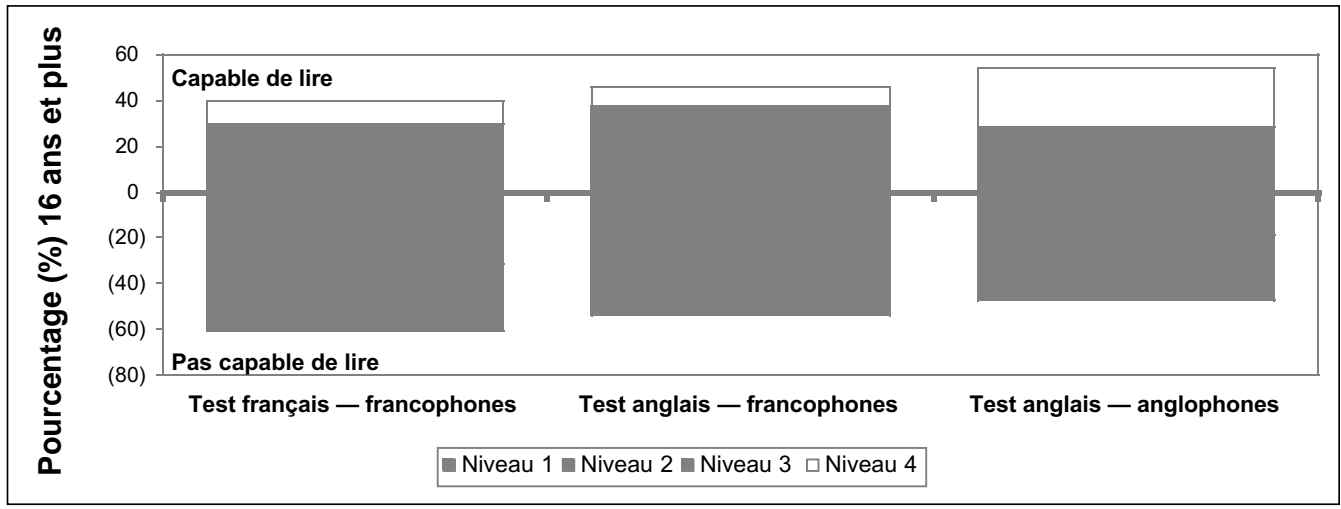

Source : Statistique C anada, R ecensement 1996, 2A , 0 A F

«Près d'un tiers de la population francophone adulte ne savait pas lire (niveau 1) et un autre $30 \%$ avait de la difficulté à lire (niveau 2). »

« II est aussi évident que les niveaux d'alphabétisme plus faibles se situent dans les régions $\mathrm{N}$ ord et Sud0 uest de la province. »
Chez les francophones, les capacités de lecture diminuent avec l'âge comme l'illustre la Figure 8. Près d'un tiers de la population francophone adulte ne savait pas lire (niveau 1) et un autre $30 \%$ avait de la difficulté à lire (niveau 2). $0 \mathrm{n}$ a également relevé des différences entre les hommes et les femmes : $44 \%$ des femmes francophones ont des capacités de lecture supérieures (niveaux 3 et 4), comparativement à $34 \%$ des hommes francophones. II est aussi évident que les niveaux d'al phabétisme plus faibles se situent dans les régions $\mathrm{N}$ ord et Sud- $\mathrm{O}$ uest de la province.

Quelles sont les conséquences d'un faible niveau d'alphabétisme? Les personnes anal phabètes ont plus tendance à être en chômage, à occuper des emplois moins bien rémunérés et à vivre dans des ménages à faible revenu (Shalla et Schellenberg, 1998). À cette situation peu réjouissante s'ajoute un autre fait troublant : «les parents et les enfants de ménages à faible revenu sont moins nombreux à participer aux activités d'amélioration des capacités de lecture que ceux qui jouissent d'un niveau de vie plus élevé » (Shalla, 1998). 


\section{Figure 8 - Répartition des capacités de lecture à l'échelle de compréhension des textes suivis, selon l'âge - population francophone}

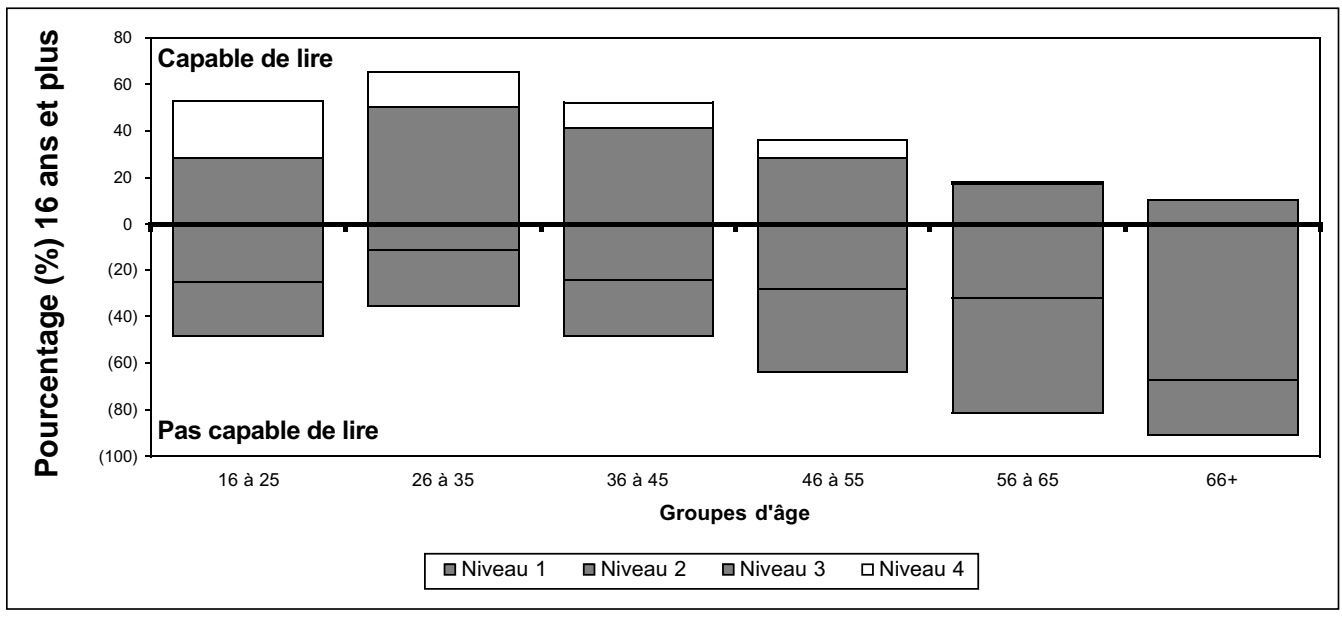

Source : Statistique C anada, R ecensement 1996, 2A , 0 A F

«... il faut... souligner l'importance de développer des habiletés de lecture auprès des francophones et assurer aux enfants francophones un accès aux activités visant à améliorer leurs capacités de lecture.»
Par ailleurs, la faiblesse descapacités de lecture peut influer sur les activités simples de la vie quotidienne. Par exemple, les adultes vivants dans des ménages à faible revenu sont «cing fois plus susceptibles d'avoir besoin d'aide pour lire desinstructions sur un contenant de médicaments »(Shalla 1998). C'est dire à quel point un faible niveau d'alphabétisme peut soulever de nombreuses difficultés pour les personnes qui vivent avec un tel déficit.

Compte tenu de l'influence d'une génération sur la suivante, il faut, sans plus tarder, souligner l'importance de développer des habiletés de lecture auprès des francophones et assurer aux enfants francophones un accès aux activités visant à améliorer leurs capacités de lecture. Dans une nouvelle économie axée sur l'information, les personnes possédant de faibles capacités de lecture éprouveront plus de difficultés à s'intégrer au marché du travail, à atteindre un bien- être économique et social et à bénéficier d'une santé optimale. Des améliorations dans le domaine de l'alphabétisme, qu'on observe déjà chez les francophones, 
permettraient aux francophones de modifier sensiblement leur situation actuelle.

\section{Participation au marché du travail}

En 1996, le taux de participation francophone au marché du travail était inférieur à celui de la province, particulièrement chez les 45 à 65 ans (Figure 9). Fait à noter, dans les groupes de moins de 35 ans, les francophones avaient un taux de participation supérieur à celui de leurs homologues de la province.

\section{Figure 9 - Participation au marché du travail, selon l'âge - francophones et population totale de l'O ntario}

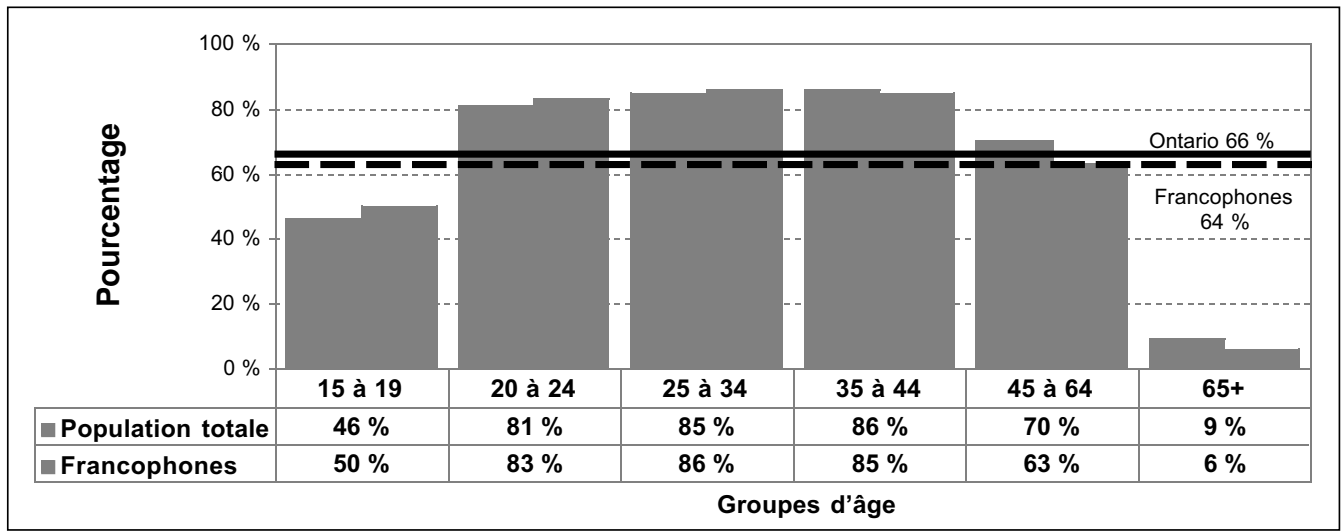

Source : Statistique C anada, R ecensement 1996, 2A , 0 A F

« L es taux de chômage étaient semblables (9\%) pour les francophones que pour l'ensemble de la population ontarienne »
O n associe le chômage à la détérioration de la santé (Zöller et Lessof 1998). Les taux de chômage étaient semblables (9 \%) pour les franco phonesque pour l'ensemble de la population ontarienne. Toutefois, on a relevé des différences entre les groupes lorsqu'on compare leshommes et lesfemmes. Alors que le taux des femmes francophones était légèrement inférieur au taux provincial $(9 \%$ par rapport à $10 \%)$, on a noté l'inverse chez les hommes francophones (10\% par rapport à $9 \%$ ). 
"L orsqu'on ajoute le facteur âge, on s'aperçoit que les groupes francophones plus jeunes (moins de 35 ans) touchent un revenu moyen supérieur à celui de l'ensemble de la population. »

\section{Revenu}

Les recherches démontrent qu'il existe un lien entre le revenu et l'état de santé (Z öller, 1998). En 1995, le revenu moyen individuel des francophones était légèrement inférieur (27044 \$) à celui de l'ensemble de la population (27 309 \$). Lorsqu' on ajoute le facteur âge, on s'aperçoit que les groupesfrancophones plusjeunes (moins de 35 ans) touchent un revenu moyen supérieur à celui de l'ensemble de la population. Toutefois, cela signifie que l'inverse est vrai pour les francophones âgés de 35 ans et plus (Figure 10).

\section{Figure 10 - Revenu total moyen par personne, selon l'âge - francophones et population totale de l'O ntario}

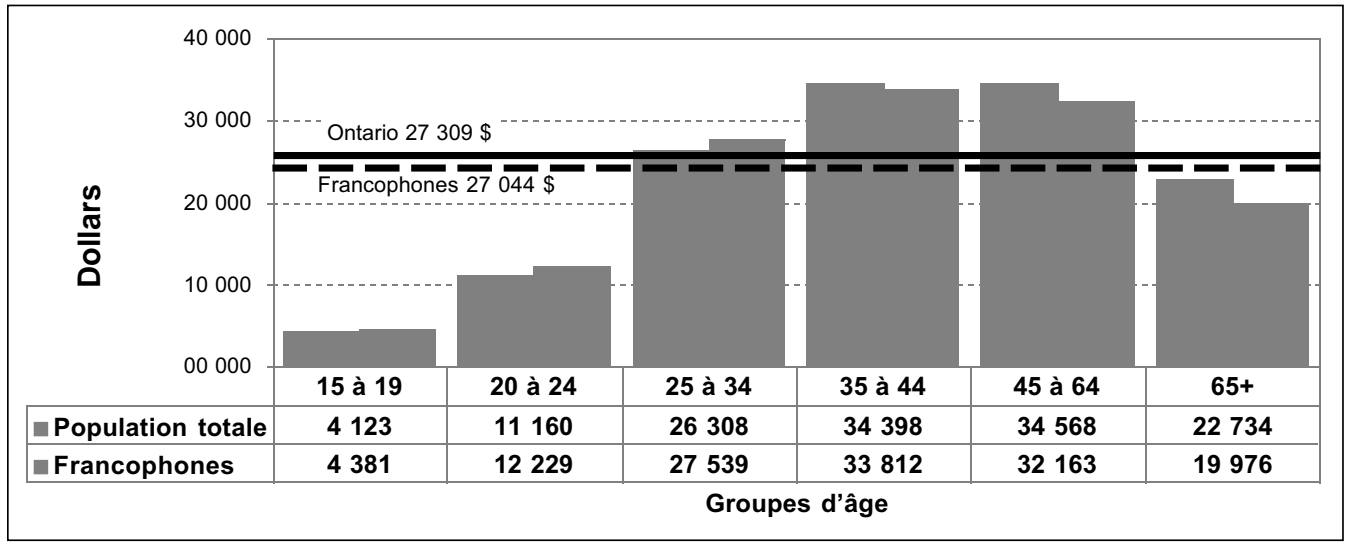

Source : Statistique C anada, R ecensement 1996, 2A , 0 A F

En 1995, le revenu moyen des femmes francophones représentait $65 \%$ du revenu moyen des hommes francophones, ce qui est légèrement mieux que le rapport pour la population totale (63\%). Les francophones avaient un revenu familial moyen inférieur à celui de la population ontarienne (61 887 \$ par rapport à 64227 \$). Ce sont les francophones du Nord-Est qui avaient le revenu familial moyen le plus bas (55 870 \$). 


\section{Seuil de faible revenu}

Le faible revenu est associé à différents problèmes de santé, tels que la naissance de bébés de faible poids, une espérance de vie plus courte, l'invalidité et diverses maladies (Comité consultatif fédéral provincial territorial sur la santé de la population 1994). Lesdonnées du recensement 1996 indiquent que les francophones sont un peu moins susceptibles de vivre sous le seuil de faible revenu. En 1996, $18 \%$ desfemmes francophones et $15 \%$ deshommesfrancophones avaient des revenus équivalents ou inférieurs aux seuils de faible revenu, comparativement à $19 \%$ des femmes et à $16 \%$ deshommes dans l'ensemble de la population de l'O ntario. O n a pu observer des différences notables en fonction de l'âge et du sexe (Figure 11).

«U ne femme

francophone sur trois âgée de 75 ans et plus vivait sous le seuil de faible revenu. $C$ e taux est près de trois fois plus élevé que celui des hommes appartenant au même groupe d'âge » U ne femme francophone sur troisâgée de 75 ans et plus vivait sous le seuil de faible revenu. C e taux est près de trois fois plusélevé que celui des hommes appartenant au même groupe d'âge. Dans l'ensemble de la population ontarienne, ce même taux était d'environ une femme sur quatre, soit approximativement un taux deux fois plus élevé que celui des hommes. En tout, $31 \%$ des femmes francophones du groupe des 20 à 24 ans vivent sous le seuil de pauvreté, comparativement à $27 \%$ desfemmes ontariennes du même groupe d'âge et à $23 \%$ des hommes francophones appartenant au même groupe d'âge.

\section{Figure 11 - Seuil de faible revenu, selon l'âge - francophones et population totale de l'Ontario}

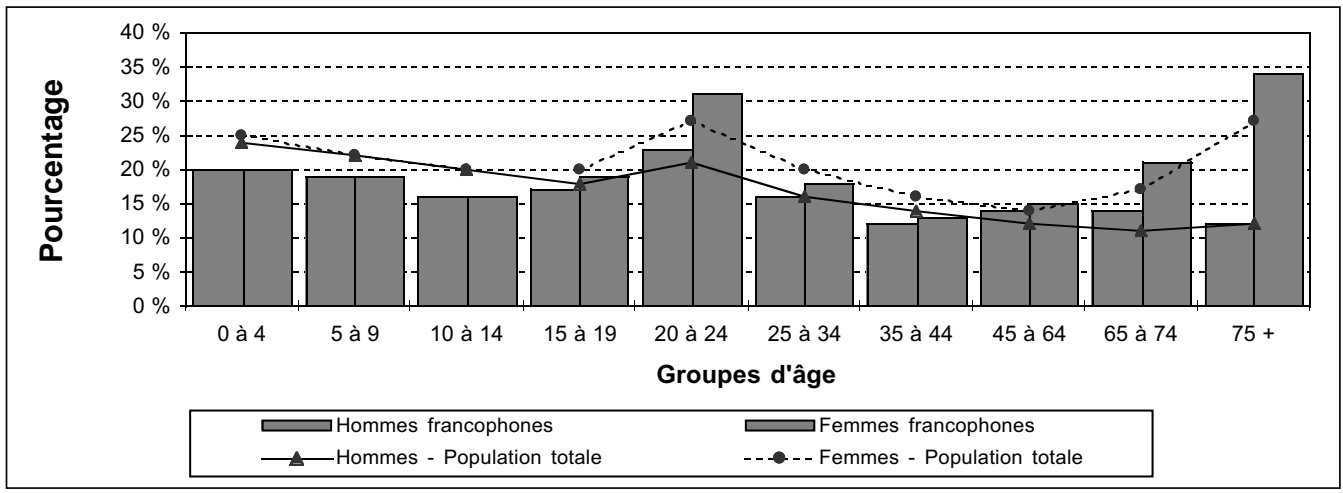

Source : Statistique C anada, R ecensement 1996, 2A , 0 A F 


\section{Structure familiale}

Certaines études sur lesfamillesfrancophones (Bernier 1997) ont identifié peu de différences entre les familles francophones et les familles ontariennes en général. C omme francophone, nous avons parfois l'impression que les familles francophones ont plus de soutien familial. Q ue nous révèle le dernier recensement?

« $0 \mathrm{n}$ a noté une plus faible proportion de familles monoparentales au sein de la population francophone.»

«... on remarque plus de variations régionales que dans la population ontarienne... le taux de familles monoparentales varie de $7 \%$ dans le $\mathrm{N}$ ord- 0 uest à $11 \%$ dans l'E st. »
On a noté une plus faible proportion de familles monoparentales au sein de la population francophone. Plusd'une famille francophone sur dix est dirigée par une seule personne, comparativement à une sur sept pour la population en général. La majorité des famillesmonoparentales est dirigée par desfemmes. C ependant, on remarque plus de variations régionales que dans la population ontarienne. $\mathrm{C}$ hez les familles francophones, le taux de familles monoparentales varie de $7 \%$ dans le $\mathrm{N}$ ord- $\mathrm{O}$ uest à $11 \%$ dans l'Est.

U ne dernière donnée au chapitre de la structure familiale mérite d'être soulignée. U n francophone sur cinq vit seul ou ne vit pas avec sa famille immédiate (enfants ou parents). À l'instar du recensement de 1991, ce taux est plus élevé chez les francophonesque le taux provincial. Le pourcentage de personnes vivant dans des ménages non familiaux s'est accru entre 1991 et 1996 et ce, tant dans la population francophone que dans l'ensemble de la population ontarienne. II est passé de $17 \%$ à $20 \%$ chez les femmes francophones, et de $15 \%$ à $19 \%$ chez les hommes francophones.

La proportion de francophones vivant seul ou n'ayant pas de lien de parenté immédiat avec les personnes partageant la même résidence ${ }^{2}$, est plus élevée chez les personnes âgées, notamment chez les femmes. C ela représente $20 \%$ des femmes francophones et $19 \%$ des hommes francophones, par rapport à $17 \%$ et $15 \%$ respectivement de la population ontarienne. Environ deux fois plus de femmes francophonesâgées 65 et plus vit dans des ménages non familiaux, comparativement aux hommes francophones. 


\section{Conclusion}

«... la population

francophone continue à être généralement plus âgée, sous-scolarisée, proportionnellement moins nombreuse sur le marché du travail et à avoir un niveau de revenu un peu plus faible.Toutefois... ces différences entre les francophones et la population ontarienne $s$ 'amenuisent sensiblement, voire disparaissent complètement lorsqu' on retient les sous-groupes les plus jeunes de cette population. »
D epuis trop longtemps, on a nourri une perception «misérabiliste » de la communauté francophone, parce qu'elle était moins scolarisée, plus pauvre, etc. II est peut-être temps de revoir cette perception à la lumière des plus récentes données. La population francophone possède certains traits distinctifs par rapport à l'ensemble de la population ontarienne, mais, en cela, elle n'est pas nécessairement défavorisée.

C e profil démographique fait ressortir plusieurs traits distinctifs qui caractérisaient la population francophone de l'O ntario.A insi, Iorsqu'on la compare à l'ensemble de la population ontarienne, la population francophone continue à être généralement plus âgée, sous-scolarisée, proportionnellement moins nombreuse sur le marché du travail et à avoir un niveau de revenu un peu plus faible.Toutefois, on constate fort heureusement que ces différences entre les francophones et la population ontarienne s'amenuisent sensiblement, voire disparaissent complètement lorsqu'on retient les sous-groupes les plus jeunes de cette population. Et il y a donc lieu d'envisager l'avenir sous des jours meilleurs.

Comme intervenants et intervenantes dans le domaine de la santé et des services sociaux, il est essentiel de bien connaitre le profil de la population francophone que nous desservons. Les modèles de promotion de la santé de la population exigent de prendre en ligne de compte l'éventail entier des déterminants de la santé et intervenir à plusieurs niveaux de la société (Santé C anada, 1996). De plus, on ne saurait dire l'importance que revêt pour la planification de programmes, le repérage de sous-populations au niveau de l'âge, au niveau régional et au niveau du genre. À la base de toute intervention, il y a un projet de connaissances auquel nous espérons avoir contribuer au moyen de ce profil démographique ${ }^{1}$. 


\section{Bibliographie}

OFFICE DES AFFAIRES FRAN COPHONES ET STATISTIQUE CANADA (1996). LeS $F$ rancophones en 0 ntario : P rofil statistique, Toronto, A uteur.

O FFICE DESAFFAIR ESFR AN C O PH O NESET STATISTIQ UE CAN ADA (1996). L es personnes âgées francophones en 0 ntario: P rofil statistique, Toronto, A uteur.

O FFICE DESAFFAIR ES FR AN COPHON ES ET STATISTIQUE CAN ADA (1996). Les jeunes francophones en 0 ntario: Profil statistique, Toronto, A uteur.

OFFICE DESAFFAIR ESFR AN CO PH O NESET STATIST IQ UE CAN ADA. (1996). L es femmes francophones en 0 ntario: Profil statistique,Toronto, A uteur.

SANTÉ CAN ADA (1996). Compte rendu de la table ronde sur la santé de la population et la promotion de la santé,A ccessible : http :/ / www.hc-sc.gc.ca/ hppb/ developpement-promotion/ pubf/ tableronde/ tableronde.htm.

SAN TÉ CAN ADA. (1996). Promotion de la santé de la population : modèle d'intégration de la santé de la population et de la promotion de la santé, Accessible : http :// ww w.hc-sc.ca/ hppb/ developpement-promotion/pub/psp/psp.htm

ZÖ LLN ER , H ., et S. LESSO F, (1998). Santé de la population : M ettre les concepts en application : R apport final, D isponible : http :// www.hc-sc.gc.ca/ hppb/ ddsp/ report-f.htm.

\section{Notes}

1. Ménages non familiaux : un ménage non familial est constitué soit d'une personne vivant seule dans un logement privé, soit d'un groupe de deux personnes ou plus qui partagent un logement privé, maisqui ne forment pas une famille de recensement. Famille de recensement : on entend par famille de recensement un couple qui est actuellement marié (avec ou sans enfants, jamais mariés) un couple vivant en union de fait (avec ou sans enfants), un parent de famille monoparentale, peu importe son état civil, qui abrite chez elle ou chez lui au moins un fils ou une fille jamais marié.

2. N ous tenons à remercier sincèrement les autres membres de l'équipe de rédaction du $R$ apport sur la santé des francophones de l'O ntario, soit D enise H ébert, R uth Sanderson, I sabelle M ichel, Alissa Palangio, Colette Fraser et aussi tous les membres du comité consultatif du rapport : Caroline Andrew, Francine D eroche, D iane Farmer, France G élinas, Jocelyne Lalonde, C arole $\mathrm{R}$ acette,V ic Sahai. M erci aussi aux membres du programme provincial de R echerche, éducation $\&$ développement en santé publique (R EDSP), tout particulièrement Ian Johnson, qui ont contribué à la publication de ce rapport. 